\title{
Skeletal muscle fibre-type shifting and metabolic profile in patients with chronic obstructive pulmonary disease
}

\author{
H.R. Gosker*, H. van Mameren\#, P.J. van Dijk", M.P.K.J. Engelen*, G.J. van der Vusse ${ }^{\star}$, \\ E.F.M. Wouters*, A.M.W.J. Schols*
}

Skeletal muscle fibre-type shifting and metabolic profile in patients with chronic obstructive pulmonary disease. H.R. Gosker, H. van Mameren, P.J. van Dijk, M.P.K.J. Engelen, G.J. van der Vusse, E.F.M. Wouters, A.M.W.J. Schols. C ERS Journals Ltd 2002.

ABSTRACT: The aim of this study was to examine the nature of fibre-type redistribution in relation to fibre metabolic profile in the vastus lateralis in chronic obstructive pulmonary disease (COPD) and COPD subtypes.

Fifteen COPD patients (eight with emphysema stratified by high-resolution computed tomography) and 15 healthy control subjects were studied. A combination of myofibrillar adenosine triphosphatase staining and immunohistochemistry was used to identify pure, as well as hybrid fibre types. For oxidative capacity, fibres were stained for cytochrome $c$ oxidase and succinate dehydrogenase activities, and glycogen phosphorylase for glycolytic capacity.

The proportion of type-I fibres in COPD patients was markedly lower $(16 \%$ versus $42 \%)$, especially in emphysema, and the proportion of hybrid fibres was higher $(29 \%$ versus $16 \%$ ) compared to controls. The proportion of fibres staining positive for oxidative enzymes was lower in COPD patients, which correlated with the proportion of type-I fibres. In COPD oxidative capacity was lower within IIA fibres.

The authors conclude that fibre-type transitions are involved in the fibre-type redistribution in chronic obstructive pulmonary disease. Low oxidative capacity is closely related to the proportion of type-I fibres, but an additional reduction of oxidative enzyme activity is present within IIA fibres. Fibre-type abnormalities may be aggravated in emphysema.

Eur Respir J 2002; 19: 617-625.
*Dept of Pulmonology, ${ }^{\#}$ Dept of Anatomy and Embryology and Dept of Physiology, University of Maastricht, Maastricht, the Netherlands.

Correspondence: H.R. Gosker

Dept of Pulmonology

Maastricht University

P.O. Box 616

6200 MD Maastricht

The Netherlands

Fax: 31433875051

E-mail: H.Gosker@pul.unimaas.nl

Keywords: Chronic obstructive, histochemistry, myosin heavy chain isoforms, oxidative capacity, pulmonary disease, skeletal muscle

Received: June 72001

Accepted after revision October 30 2001

This study was supported by a grant from the Netherlands Asthma Foundation (project number 96.16).
One of the hallmarks in chronic obstructive pulmonary disease (COPD) patients is a progressively reduced exercise capacity. Besides lung failure, skeletal-muscle dysfunction also contributes to exercise intolerance $[1,2]$. Both biochemical and morphological changes in peripheral skeletal muscles of COPD patients have been described [3], but it is unclear how these alterations develop. Abnormal fibre-type proportions have been found in the quadriceps femoris in COPD [4-6], but in these studies fibres were only classified as either type I, IIA or IIX on the basis of myofibrillar adenosine triphosphatase (mATPase) staining. In the current study, mATPase and immunohistochemical identification of fibres were combined, which allowed detection of hybrid fibres (fibres expressing multiple myosin heavy chain (MyHC) isoforms), providing useful information on the mechanism underlying fibre-type redistribution in COPD.

Data with respect to metabolic enzyme activities in the quadriceps femoris in COPD are not consistent. Glycolytic enzyme activity may or may not be increased [7, 8] and oxidative enzyme activities are reduced [7, 8]. However, SAUleda et al. [9] found that the activity of cytochrome $c$ oxidase (COX), an enzyme involved in the oxidative energy metabolism, was increased. Therefore, the enzyme profile within the identified fibre-type categories has been studied. $\mathrm{COX}$ is a mitochondrial enzyme involved in oxidative phosphorylation. Succinate dehydrogenase (SDH) is also a mitochondrial enzyme and exerts its catalytic action in the citric acid cycle. Glycogen phosphorylase (GlyP) is involved in glycogenolysis and mobilizes glucose-1-phosphate from glycogen storage for glycolysis. Hence, COX and SDH were used as markers for oxidative energy metabolism and GlyP was used as a marker for glycolytic energy metabolism in this study. With this approach it is also possible to establish to what extent changes in fibre-type proportions and enzyme activities are coupled.

Using gel electrophoresis techniques a lower type-I and a higher type-II MyHC isoform content was found in the quadriceps femoris of COPD patients compared to healthy subjects $[10,11]$. In these studies the MyHC type-I content was positively related to the carbon dioxide diffusing capacity of the lung $(D \mathrm{~L}, \mathrm{CO})$ and the forced expiratory volume in one second (FEV1). On the one hand, the relationship with $D \mathrm{~L}, \mathrm{CO}$ suggests that the loss of type-I fibres is more pronounced in patients with emphysema and on the other 
hand, the relationship with FEV1 suggests that the loss is more pronounced in patients with severe COPD. In the current study, a further insight into muscular changes in patients with and without emphysema stratified by high-resolution computed tomography (HRCT) is provided.

The aims of this study were: 1) establish whether fibre-type redistribution in COPD occurs through gradual shifts in intracellular $\mathrm{MyHC}$ isoform content, which would result in a greater proportion of hybrid fibre types in patients; 2) provide further insight into the behaviour of metabolic enzymes (oxidative and glycolytic) and their relationship to the fibre-type distribution; 3) establish if abnormal fibre-type distribution is aggravated in emphysema patients compared to nonemphysema patients.

\section{Materials and methods}

\section{Study population}

A group of 15 patients with moderate-to-severe airflow obstruction and 15 healthy age-matched volunteers were studied (table 1). All patients had COPD according to American Thoracic Society (ATS) guidelines [12] and chronic airflow limitation, defined as measured FEV $1<70 \%$ of reference FEV1. Patients also had irreversible obstructive airway disease $(<10 \%$ improvement of FEV1 predicted baseline after $\beta_{2}$-agonist inhalation). They were in a clinically stable condition and had not suffered from respiratory tract infection or exacerbation of their disease at least 4 weeks prior to the study. Medication usage and doses were retrieved from available hospital files and the referring physician. Patients received standard optimized inhalation treatment. Due to the potentially adverse effects of oral glucocorticoids on skeletal muscle, their influence on the findings in this study was specifically investigated. Doses were expressed as hydrocortisone equivalents $\cdot$ day $^{-1}$. At the time of biopsy seven of the 15 COPD patients

Table 1. - Subject characteristics and lung function data

\begin{tabular}{lcccc}
\hline & Controls & COPD & Emph- & Emph+ \\
\hline $\begin{array}{l}\text { Subjects } \\
\text { (F:M) n }\end{array}$ & $15(2: 13)$ & $15(3: 12)$ & $7(3: 4)$ & $8(0: 8)$ \\
Age yrs & $64 \pm 3$ & $67 \pm 9$ & $64 \pm 12$ & $69 \pm 4$ \\
BMI kg·m ${ }^{-2}$ & $26.7 \pm 3.0$ & $23.9 \pm 4.0^{*}$ & $25.3 \pm 2.8$ & $22.6 \pm 4.4$ \\
$\mathrm{FEV} 1 \%$ pred & $108 \pm 18$ & $42 \pm 14^{* * *}$ & $57 \pm 15$ & $32 \pm 7^{*}$ \\
$P \mathrm{a}, \mathrm{O}_{2} \mathrm{kPa}$ & $11.8 \pm 1.7$ & $9.9 \pm 1.2^{* *}$ & $10.6 \pm 1.2$ & $9.2 \pm 0.8^{*}$ \\
$D \mathrm{~L}, \mathrm{CO} \%$ pred & $121 \pm 23$ & $63 \pm 24^{* * *}$ & $84 \pm 14$ & $43 \pm 8^{* * *}$ \\
$\mathrm{RV} \%$ pred & $114 \pm 15$ & $167 \pm 32^{* * *}$ & $149 \pm 22$ & $178 \pm 39$ \\
ITGV \% pred & $107 \pm 18$ & $144 \pm 22^{* * *}$ & $125 \pm 15$ & $156 \pm 22^{*}$ \\
\hline
\end{tabular}

Data are presented as mean \pm SD. BMI: body mass index; DL,CO: diffusion capacity for carbon monoxide; FEV1: forced expiratory volume in one second; RV: residual volume; ITGV: intrathoracic gas volume; COPD: chronic obstructive pulmonary disease; Emph-: nonemphysema subgroup; Emph+: emphysema subgroup; Significance of difference compared to controls: $*: \mathrm{p}<0.05, * *: \mathrm{p}<0.01, * * *$ : $\mathrm{p}<0.001$. were on a maintenance dose of prednisolone (range 5-10 mg $\left.\cdot \mathrm{day}^{-1}\right)$. Exclusion criteria were malignancy, cardiac failure, distal arteriopathy, recent surgery, $\alpha 1$-antiprotease deficiency, severe endocrine, hepatic or renal disorder and use of anticoagulant medication. Written informed consent was obtained from all subjects and the study was approved by the medical ethical committee of the University Hospital Maastricht (Maastricht, the Netherlands).

\section{Pulmonary function tests}

All patients and control subjects underwent spirometry to determine, amongst others, FEV1, with the highest value from at least three technically acceptable assessments being used. Residual volume (RV) and intrathoracic gas volume (ITGV) were assessed by whole-body plethysmography and $D \mathrm{~L}, \mathrm{CO}$ was measured by using the single-breath method (Masterlab, Jaeger, Wurzburg, Germany). All values obtained were related to a reference value and expressed as percentage of predicted [13].

\section{Assessment of emphysema}

Evaluation of the presence of parenchymal destruction, the hallmark of emphysema [14], was performed by HRCT as described previously [15]. The severity and extent of emphysema in each scan were visually scored by two independent observers according to the direct observational method developed by SAKAI et al. [16]. HRCT scores ranged from 0 (no emphysema) to 120 (severe emphysema). Patients were stratified by HRCT score into two groups: HRCT score <30: nonemphysema subgroup (Emph-); HRCT score $>30$ : emphysema subgroup $(\mathrm{Emph}+)$.

\section{Tissue collection and processing}

Arterial oxygen tension $\left(\mathrm{Pa}_{\mathrm{a}} \mathrm{O}_{2}\right)$ was determined (ABL 330; Radiometer, Copenhagen, Denmark) in a blood sample obtained by puncture of the radial artery while breathing room air. Postabsorptive muscle biopsies of the lateral part of the quadriceps femoris were obtained under local anaesthesia by the needle biopsy technique [17]. Biopsies were placed in a drop of Tissue-tek $\mathbb{R}$ (OCT compound) on a piece of cork with the fibre orientation perpendicular to the plane of the cork. The specimen was frozen in melting isopentane, precooled in liquid nitrogen and stored at $-35^{\circ} \mathrm{C}$. Serial cryostat cross-sections $(10 \mu \mathrm{m})$ were made on a cryostat microtome at $-20^{\circ} \mathrm{C}$ and mounted on slides which were stored at $-35^{\circ} \mathrm{C}$ until analysis. Nine consecutive slides (each carrying two or three cross-sections) per biopsy sample were used for fibretype characterization and enzyme-activity staining. At least 100 (but up to 200) fibres co-existing on all nine slides were numbered and analysed as described previously [18]. Fibres were included if they were part of a cluster of $\geqslant 30$ fibres and excluded if disturbing 
artefacts were present. All staining intensities were analysed by the same person.

\section{Fibre-type characterization}

The mATPase acidic pre-incubation was performed at $\mathrm{pH} 4.4$ [19] and the mATPase alkali pre-incubation was performed at $\mathrm{pH} 10.4$ [20]. The double preincubation method was performed at $\mathrm{pH} 10.4$ and $\mathrm{pH} 4.6$ [21], the only difference being that the fixation of sections was performed prior to the alkaline pre-incubation. After pre-incubation, sections were stained and mounted [22]. Figure 1a-c show examples of mATPase staining.

Immunohistochemistry was performed with a panel of monoclonal antibodies (MAbs); anti-type I MyHC (MAb 219-1D1), anti-type IIA MyHC (MAb 333-7H1), anti-types IIA+IIX MyHC (MAb 332-3D4) [23]. Figure 1d-f show examples of MyHC staining.

Fibres were assigned to categories, first on the basis
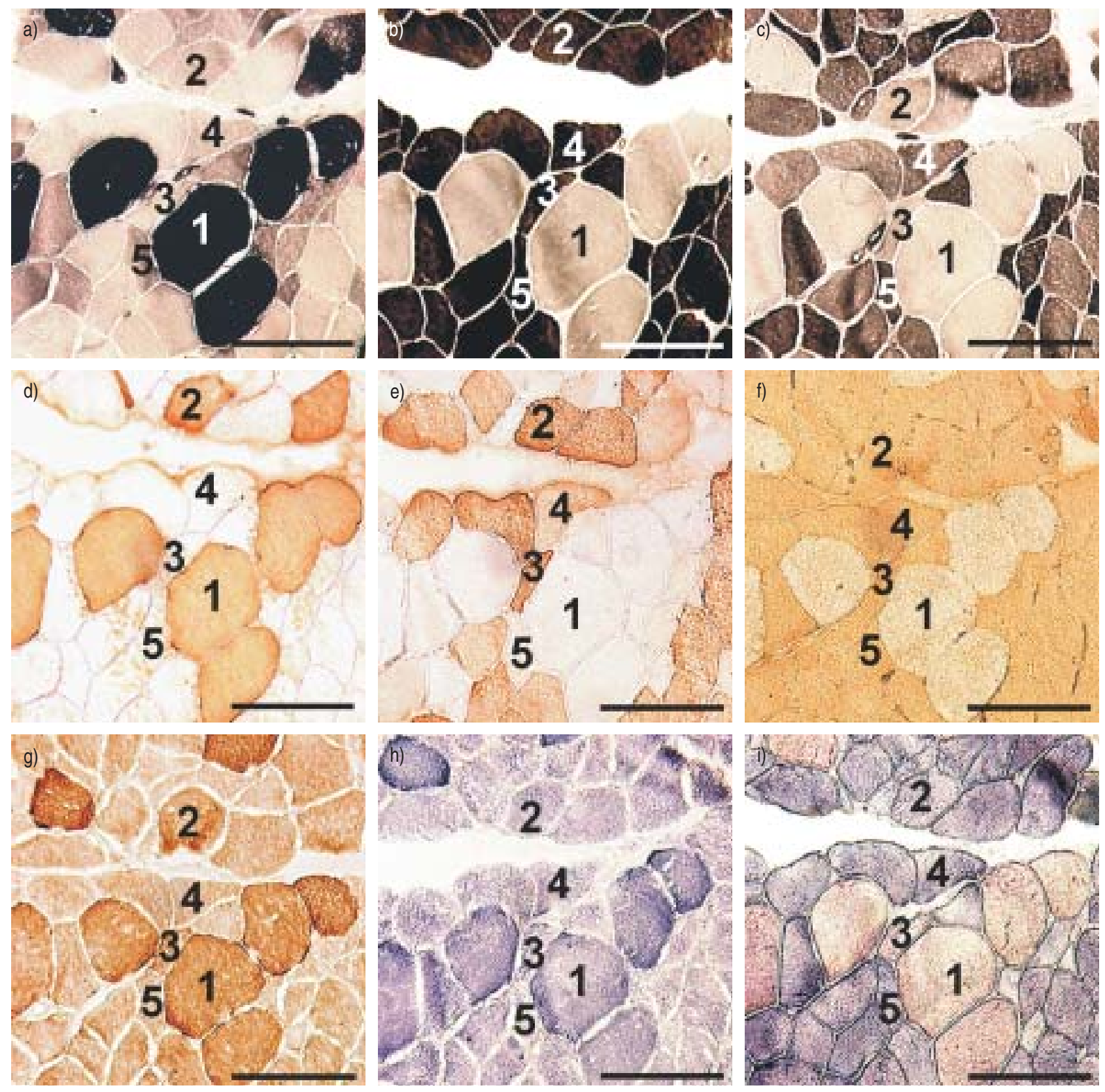

Fig. 1.-Examples of histochemical analysis in nine consecutive cross-sections. Myofibrillar adenosine trisphosphatase (mATPase) staining with: a) acidic, b) alkaline and c) double pre-incubation (positive fibres stain dark); immunohistochemistry with: d) anti-type I, e) antitype IIA and f) anti-types IIA+IIX (positive fibres stain dark). Fibres numbered 1-5 are assigned to the I, I/IIA, IIA, IIA/IIX and IIX combination of mATPase and myosin heavy chain fibre type categories, respectively (staining intensities are given in table 2). g) Cytochrome $c$ oxidase activity staining (positive fibres are coloured brown). h) Succinate dehydrogenase activity (positive fibres are coloured blue). i) Glycogen phosphorylase positive fibres are coloured purple and negative fibres yellow. Internal scale bars $=150 \mu \mathrm{m}$. 
of three mATPase staining intensities $(-,+$ or ++$)$ and secondly by immunoreactivity $(-$ or + ) (table 2$)$. The following combination of mATPase and MyHC (CMM) fibre-type categories were made: type I, I/IIA, IIA, IIA/IIX, IIX and miscellaneous (misc).

\section{Enzyme activity staining}

COX staining was performed by incubating sections for $1 \mathrm{~h}$ in a $50 \mathrm{mM}$ Tris/ $\mathrm{HCl}$ buffer $(\mathrm{pH}$ 7.6) contain-

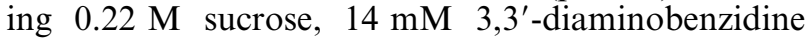
tetrahydrochloride, $80 \mu \mathrm{M}$ cytochrome c and $1300 \mathrm{U}$ catalase. Sites of COX activity were coloured brown [24]. SDH staining was performed by incubating sections for $1 \mathrm{~h}$ at $37^{\circ} \mathrm{C}$ in a $0.2 \mathrm{M}$ sodium phosphate buffer containing $0.1 \mathrm{M}$ succinic acid and $1.2 \mathrm{mM}$ nitro-blue tetrazolium. Sites of SDH activity were coloured blue [25]. GlyP staining was performed by incubating sections for $5 \mathrm{~min}$ in a $43 \mathrm{mM}$ sodium acetate buffer (pH 5.6) containing $7 \mathrm{mM}$ glucose-1phosphate, $1 \mathrm{mM}$ adenosine monophosphate (AMP), $0.01 \%$ glycogen and $15 \%$ ethanol [25], after which the newly formed polysaccharide was coloured blue with Lugol's iodine. COX and SDH cross-sections were dehydrated in an ethanol range and mounted in

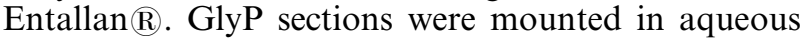
glycerin/gelatine that had been coloured with Lugol's iodine. Within each section, three staining intensities were chosen reflecting fibre enzyme activity: positive $(+)$, negative $(-)$ or intermediate $( \pm)$. For example, a fibre positive for $\mathrm{COX}$, intermediate for SDH and negative for GlyP would be $\mathrm{COX}+, \mathrm{SDH} \pm$ and GlyP-. Figure 1g-i show examples of enzyme activity staining.

\section{Statistical analysis}

Values are reported as mean \pm standard deviation (SD) and error bars in figures reflect standard error of the mean (SEM). Intergroup data were compared using an unpaired t-test (corrected for unequal variances if appropriate) or one-way analysis of variance (ANOVA) (with unpaired t-test as post hoc test).

Table 2. - Fibre-type classification

\begin{tabular}{|c|c|c|c|c|c|c|}
\hline \multirow{2}{*}{$\begin{array}{l}\text { CMM } \\
\text { fibre } \\
\text { type }\end{array}$} & \multicolumn{3}{|c|}{ mATPase } & \multicolumn{3}{|c|}{ МyHC } \\
\hline & pH 4.4 & $\mathrm{pH} 10.4$ & Double & I & IIA & IIAX \\
\hline I & ++ & - & - & + & + or - & + or - \\
\hline I/IIA & $\begin{array}{c}++ \\
+ \text { or }++\end{array}$ & $+\underset{+}{\text { or }++}$ & $\begin{array}{l}- \\
-\end{array}$ & $\begin{array}{l}+ \\
+\end{array}$ & $\begin{array}{l}+ \\
+\end{array}$ & $\begin{array}{l}+ \\
+\end{array}$ \\
\hline IIA & $\begin{array}{c}701 \\
-\end{array}$ & $\begin{array}{r}+1 \\
++\end{array}$ & - & + or - & $\begin{array}{l}+ \\
+\end{array}$ & $\begin{array}{l}+ \\
+\end{array}$ \\
\hline IIA/IIX & - or + & ++ & + or ++ & - & + & + \\
\hline IIX & + & ++ & + or ++ & - & - & + \\
\hline Misc & $\begin{array}{c}++ \\
+ \text { or }++\end{array}$ & $\begin{array}{c}++ \\
+ \text { or }++\end{array}$ & $\begin{array}{c}+ \text { or } \\
-\end{array}$ & $\begin{array}{l}+ \\
+\end{array}$ & $\begin{array}{l}+ \\
-\end{array}$ & $\begin{array}{l}+ \\
+\end{array}$ \\
\hline
\end{tabular}

Fibres were classified first on the basis of myofibrillar adenosine trisphosphatase (mATPase) staining intensities $(-,+$ or ++$)$ and further classified by immunoreactivity (- or + ); MyHC: myosin heavy chain; CMM: the combination of mATPase and MyHC fibre-type categories.
Potential relationships between variables of interest were evaluated using the Pearson correlation test or a partial correlation to correct for age. Data were analysed according to the guidelines of Altman et al. [26]. A two-tailed $\mathrm{p}<0.05$ was considered statistically significant.

\section{Results}

Fifteen COPD patients (12 males and three females) and fifteen healthy controls (13 males and two females) participated in this study (table 1). There were no differences in sex or age between the groups. The body mass index (BMI), $\mathrm{Pa}_{2} \mathrm{O}_{2}, \mathrm{FEV} 1$ and the $D$ L,CO were significantly lower, whereas the RV and the ITGV were higher in patients than in controls. Eight patients (with HRCT scores $>30$ ) were assigned to Emph+ and seven patients (with HRCT scores $<30$ ) to the Emph- subgroup. The $P \mathrm{a}, \mathrm{O}_{2}, \mathrm{FEV} 1$ and the $D \mathrm{~L}, \mathrm{CO}$ were lower and the RV and the ITGV were higher for the Emph+ than for the Emph- COPD subtype.

A typical example of histochemical results is shown in figure 1. First the data was interpreted as if based purely on mATPase staining (fig. 2a). The proportion of mATPase type-I fibres was lower $(19 \%$ versus
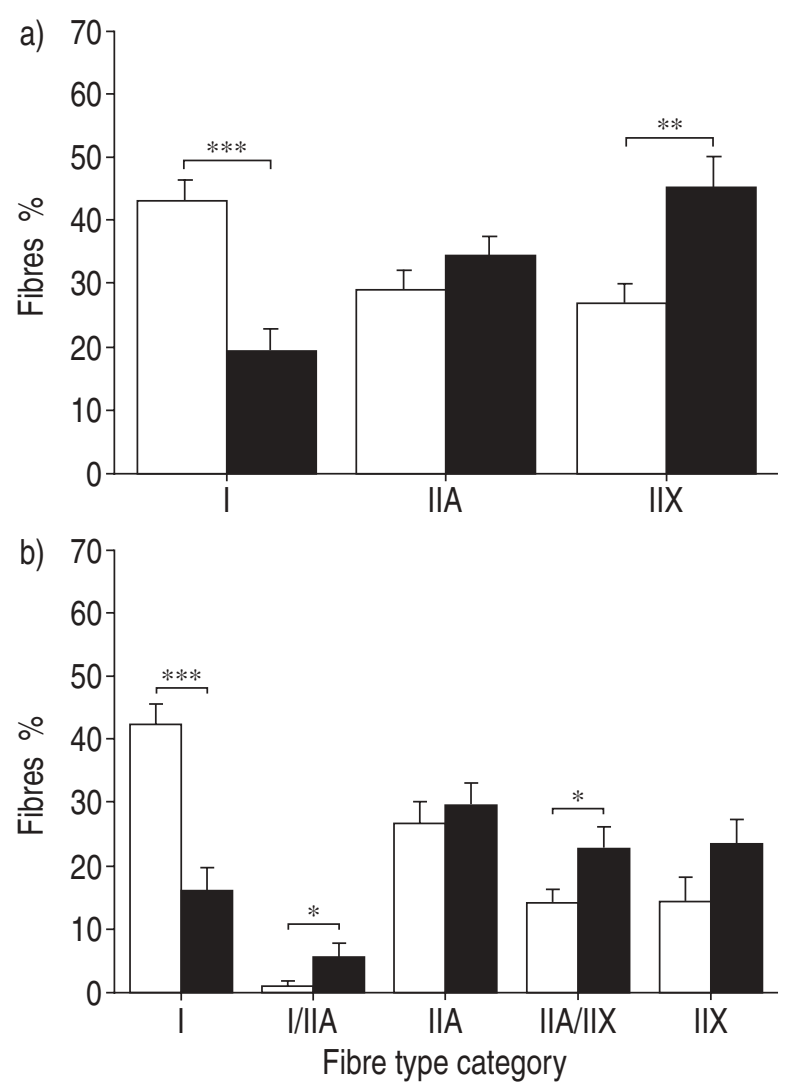

Fig. 2.-a) Myofibrillar adenosine trisphosphatase (mATPase) and b) combination of mATPase and myosin heavy chain fibre-type categories in the vastus lateralis. $\square$ : controls; $\mathbf{0}$ : chronic obstructive pulmonary disease patients. Significance of difference between the groups: $*: \mathrm{p}<0.05 ;{ }^{* *}: \mathrm{p}<0.01 ;{ }^{* * *}: \mathrm{p}<0.001$. 
$43 \%, \mathrm{p}<0.001)$ and the proportion of mATPase typeIIX fibres was higher ( $46 \%$ versus $27 \%, p=0.004)$ in the COPD group compared to controls. There was no difference in the percentage of mATPase typeIIA fibres. By combining the three mATPase preincubation protocols with immunohistochemistry, five fibre-type categories were defined: CMM type I, IIA, IIX, I/IIA and IIA/IIX fibres, the latter two representing hybrid fibres (table 2). Fibre-type distribution was subsequently re-established with this more accurate method (fig. 2b). Again, the proportion of type-I fibres was lower $(16.1 \%$ versus $42.4 \%, \mathrm{p}<0.001)$ and the percentage of type-IIX fibres tended to be higher $(23.8 \%$ versus $14.7 \%, \mathrm{p}=0.088)$ in COPD than in controls. In addition, the proportion of hybrid-fibres I/IIA (5.9\% versus $1.3 \%, \mathrm{p}=0.044)$, IIA/IIX $(22.9 \%$ versus $14.4 \%, \mathrm{p}=0.041)$, and total (I/IIA+IIA/IIX) (28.8\% versus $15.7 \%, \mathrm{p}=0.008)$ were higher in COPD compared to healthy subjects. Fibres that did not fit into one of the categories discussed earlier were assigned to the miscellaneous group. Although the percentage of these fibres was also higher in COPD than in controls $(1.6 \%$ versus $0.2 \%, \mathrm{p}=0.017)$, their overall contribution to fibre-type composition was negligible. There was no difference in the percentage of type-IIA fibres between both groups.

Figure 3 shows the results of enzyme activity staining of $\mathrm{COX}+, \mathrm{SDH}+$ and GlyP+. Overall, the proportion of $\mathrm{COX}+$ and $\mathrm{SDH}+$ fibres was lower $(20.1 \%$ versus $45.8 \%, \mathrm{p}<0.001$ and $14.2 \%$ versus $36.3 \%, \mathrm{p}<$ 0.001 ), whereas the proportion of $\mathrm{COX}-$ and $\mathrm{SDH}+$ fibres was higher $(35.3 \%$ versus $23.8 \%, p=0.003$ and $47.9 \%$ versus $25.0 \%, \mathrm{p}<0.001$, data not shown) in COPD than in the control group. The percentage of $\mathrm{COX} \pm$ fibres was also higher in COPD than in controls $(44.6 \%$ versus $30.3 \%, \mathrm{p}=0.002$, data not shown). There were no differences in the proportion of GlyP+, GlyP-, GlyP \pm and $\mathrm{SDH} \pm$ fibres between patients and controls. The proportion of GlyP+ fibres in patients not treated with glucocorticosteroids $(45.8 \%)$ was higher than in controls $(34.2 \%, \mathrm{p}=0.045)$ and also

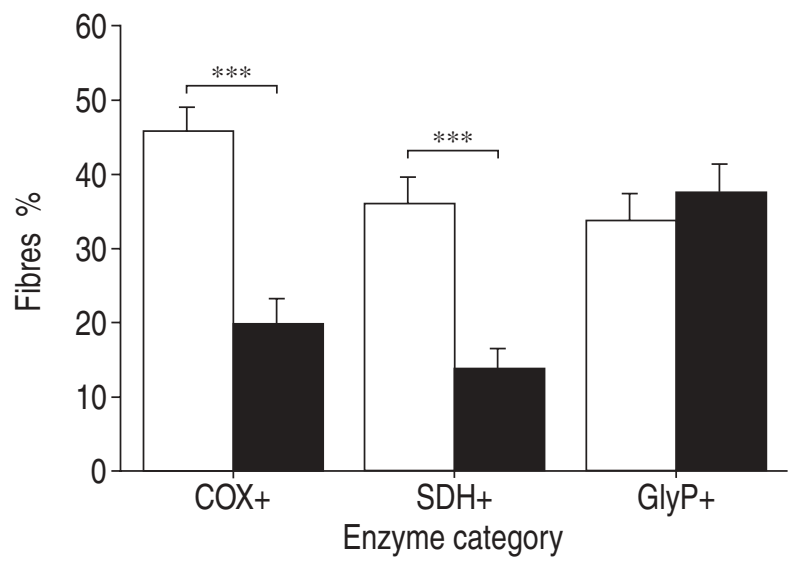

Fig. 3.-Enzyme activity staining in the vastus lateralis. COX: cytochrome $c$ oxidase; SDH: succinate dehydrogenase; GlyP: glycogen phosphorylase. $\square$ : controls; $\square$ : chronic obstructive disease (COPD) patients. Significance of difference between the groups: $* *: \mathrm{p}<0.01 ; * * *: \mathrm{p}<0.001$.
Table 3. - Proportions of cytochrome $c$ oxidase (COX+) and succinate dehydrogenase $(\mathrm{SDH}+)$ fibres within the combination of myofibrillar adenosine trisphosphatase and myosin heavy chain fibre type categories

\begin{tabular}{lcclcc}
\hline \multirow{2}{*}{ Fibre type } & \multicolumn{2}{c}{$\mathrm{COX}+$ fibres \% } & & \multicolumn{2}{c}{$\mathrm{SDH}+$ fibres \% } \\
\cline { 2 - 3 } \cline { 6 - 6 } & Controls & COPD & & Controls & COPD \\
& & & & & \\
\hline I & 87.8 & 82.7 & & 81.4 & 76.8 \\
I/IIA & 73.3 & $33.3^{*}$ & & 56.0 & $19.9^{*}$ \\
IIA & 34.1 & $7.8^{* *}$ & & 10.2 & $1.6^{*}$ \\
IIA/IIX & 2.5 & 2.4 & & 0.7 & 1 \\
IIX & 0 & 0.4 & & 0 & 0.1 \\
\hline
\end{tabular}

Significance of difference compared to controls. *: $\mathrm{p}<0.05$; $* *: \mathrm{p}<0.01$.

higher than in patients who did receive glucocorticosteroids $(29.2 \%, \mathrm{p}=0.017)$. Since enzyme activity staining and fibre-type characterization were performed on serial cross-sections, enzyme activity could be evaluated within fibre-type categories. In the CMM fibretype categories I/IIA and IIA COPD patients had a lower proportion of fibres positive for the oxidative enzymes, as shown for $\mathrm{COX}+$ and $\mathrm{SDH}+($ table 3$)$. In CMM fibre-type I/IIA the proportion of COX \pm fibres was higher for patients (data not shown).

Within COPD there was a more pronounced decrease in the percentage of CMM type-I fibres in Emph+ than in Emph- (10.0\% versus $23.1 \%, \mathrm{p}=0.045)$ compared to controls (fig. 4). In each group at least one patient with no CMM type-I fibres at all, was found. No differences between the two subtypes were observed with respect to enzyme activity staining.

Strong relationships were found for COPD between the proportion of CMM type-I fibres and the proportions of $\mathrm{COX}+(\mathrm{r}=0.77, \mathrm{p}=0.001)$ and $\mathrm{SDH}+$ $(r=0.84, p<0.001)$ fibres (fig. 5). A positive relationship between age and the percentage of type-I fibres in healthy controls $(\mathrm{r}=0.61 ; \mathrm{p}=0.015)$ was also observed. This relationship was also seen in the Emph- COPD subtype, although this did not reach statistical

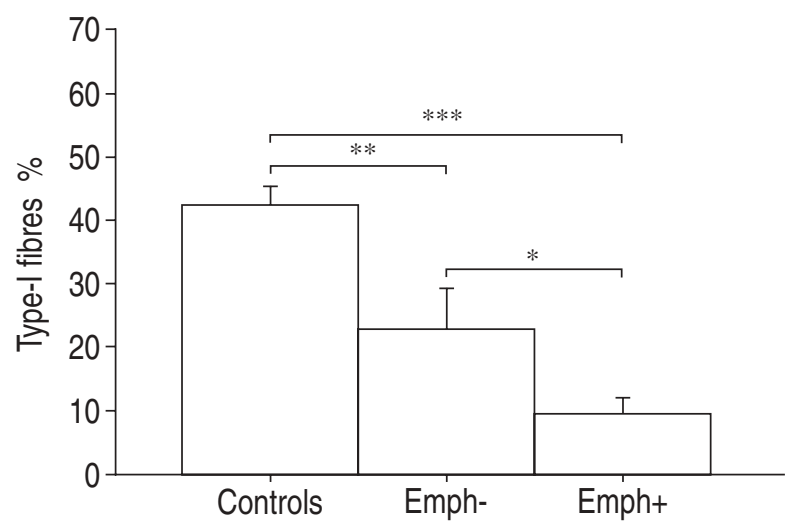

Fig. 4.-Combination of myofibrillar adenosine trisphosphatase and myosin heavy chain fibre type-I proportion in chronic obstructive pulmonary disease subgroups. Emph-: nonemphysema; Emph+: emphysema. Significance of difference between the groups: $*: \mathrm{p}<0.05 ; * *: \mathrm{p}<0.01$. 


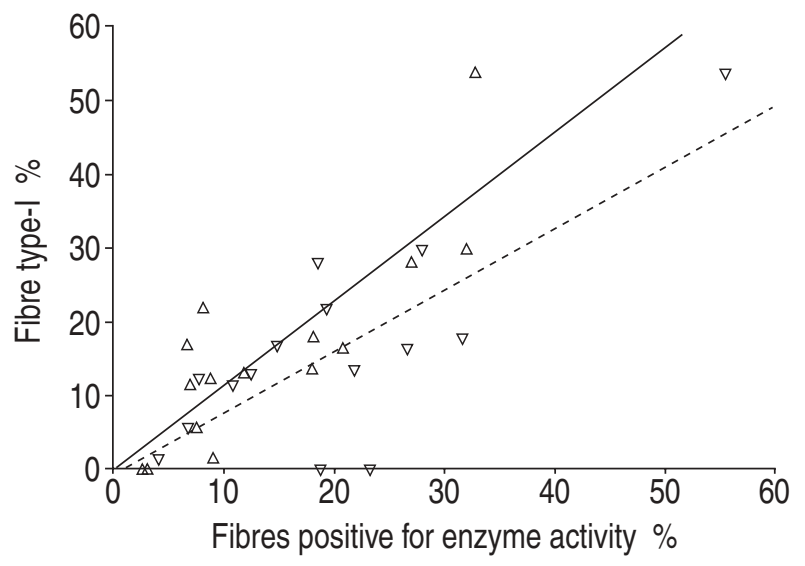

Fig. 5.-Relationship between the combination of myofibrillar adenosine trisphosphatase and myosin heavy chain fibre type-I proportion and oxidative capacity in chronic obstructive pulmonary disease patients. $\triangle$ and -: Succinate dehydrogenase $(\mathrm{r}=0.84, \mathrm{p}<0.001) ; \nabla$ and - - : cytochrome c oxidase $(\mathrm{r}=0.77$, $\mathrm{p}=0.001)$.

significance $(\mathrm{r}=0.73 ; \mathrm{p}=0.061)$. This relationship was absent in Emph+ (r=0.49; $\mathrm{p}=0.290)$. No correlations between fibre-type proportions and lung function data were found. The proportion of $\mathrm{COX}+$ fibres positively correlated with the $\mathrm{Pa}_{\mathrm{a}} \mathrm{O}_{2}(\mathrm{r}=0.60, \mathrm{p}=0.019)$.

\section{Discussion}

This study strongly suggests that the I to IIX fibretype redistribution in the vastus lateralis of COPD patients occurs through gradual shifts in intracellular $\mathrm{MyHC}$ isoform content. Accordingly, the proportions of fibres with high activities of enzymes involved in oxidative energy metabolism are reduced in patients. These proportions are closely related to the proportion of type-I fibres, but even within IIA fibres oxidative capacity is reduced in COPD. There were no differences in glycolytic capacity between patients and controls. Furthermore, for the first time, it was shown that the low fibre type-I proportion is aggravated in patients with emphysema compared to COPD patients without emphysema.

In the present study, individual fibres were identified on the basis of a combination of three different mATPase staining techniques and immunohistochemistry. By doing this, the accurate distinction of type I, IIA and IIX fibres, but also I/IIA and IIA/IIX hybrid fibres could be made. Hybrid fibres contain multiple $\mathrm{MyHC}$ isoforms, which can co-exist in any ratio. In the present study, fibres were identified as being hybrid only if indicated by mATPase staining and immunohistochemistry. The higher percentage of hybrid fibres in COPD strongly suggests that gradual transformation of one fibre type into another takes place: $\mathrm{I} \rightarrow \mathrm{I} / \mathrm{IIA} \rightarrow \mathrm{IIA} \rightarrow$ IIA/IIX $\rightarrow$ IIX.

Unlike the mATPase type-IIX fibre-type proportion, the difference in CMM fibre type-IIX proportion between COPD patients and controls did not reach statistical significance. This can be explained by the fact that part of the mATPase IIX fibres are now assigned to the hybrid IIA/IIX fibre-type category. Compared to healthy subjects, a reduced proportion of mATPase type-I fibres and an increased percentage of mATPase type-II (IIA and/or IIX) fibres have been previously reported in COPD [4-6]. However, large variations in fibre-type distribution exist between these studies. Differences in disease severity and study populations may explain these differences in part, but the large diversity in applied histochemical techniques is also a contributing factor. In addition, hybrid fibres were formerly not often detected and therefore misclassified. For example, hybrid I/IIA fibres stain intermediate for mATPase activity after pre-incubation at $\mathrm{pH} 4.4$ and will be identified as IIX fibres.

In this study, the proportions of $\mathrm{COX}+$ and $\mathrm{SDH}+$ fibres were reduced in the COPD group compared to controls, while the percentages of COX- and SDHfibres were increased. Although enzyme activity staining is a semiquantitative method, it provides useful information with respect to localization. Accordingly, in muscle biopsy specimens, reduced activities of the mitochondrial enzymes citrate synthase, SDH and hydroxyacyl-coenzymeA dehydrogenase have been reported in COPD [7, 8]. The present data are not consistent with the outcome of the study performed by SAULEDA et al. [9], who found an increased COX activity and a negative correlation between COX activity and the $\mathrm{Pa}_{\mathrm{a}} \mathrm{O}_{2}$ for $\mathrm{COPD}$ patients. Since $\mathrm{COX}$ is involved in oxidative phosphorylation and it is the final enzyme of the respiratory chain interacting with oxygen, the authors have previously suggested that its increased activity may enhance the efficiency of residual oxygen extraction as an adaptation to hypoxia [3]. In contrast, a decrease in the percentage of $\mathrm{COX}+$ fibres and a positive correlation between the proportion of $\mathrm{COX}+$ fibres and the $\mathrm{Pa}, \mathrm{O}_{2}$ was observed in this study. Various explanations can be offered for the discrepancy between this data and previously published reports [9]. First, a point of consideration is that the mean $\mathrm{Pa}_{2} \mathrm{O}_{2}$ in this patient group was not as low as the $P \mathrm{a}, \mathrm{O}_{2}$ found by SAULEDA et al. [9] (9.9 $\mathrm{kPa}$ versus $8.3 \mathrm{kPa}$ respectively). If hypoxia could upregulate COX activity, this would imply that the COX activity in the patient group in this study is lower than the COX activity in the COPD group observed by SAuleda et al. [9]. Second, one could also argue that despite the reduced proportion of $\mathrm{COX}+$ fibres, overall COX activity is elevated due to the observed increased proportion of $\mathrm{COX} \pm$ fibres in patients. However, overall staining intensity for COX was certainly not higher in COPD sections compared to controls. In addition, there was no relationship between the proportion of $\mathrm{COX} \pm$ fibres and the $\mathrm{Pa}, \mathrm{O}_{2}$. More research is needed to clarify the behaviour of COX in skeletal muscle of COPD.

One study described increased glycolytic enzyme activity phosphofructokinase (PFK) in the vastus lateralis of COPD patients [8]. In addition, increased PFK activity has been reported in lung transplant recipients compared to controls, although GlyP activity was found to be unchanged [27]. Correspondingly, an increased percentage of GlyP+ fibres was not 
found in this study. An explanation for the unchanged $\mathrm{GlyP}+$ fibre proportion in the present study is that seven from the 15 COPD patients received low doses of oral glucocorticosteroids. The proportion of $\mathrm{GlyP}+$ fibres in patients not receiving corticosteroids was higher than in controls, suggesting that corticosteroids adversely affect GlyP activity. However, patients frequently suffering from exacerbations are more likely to receive corticosteroids and therefore systemic inflammation may be involved as well. The effect of glucocorticosteroids on glycolytic metabolism needs to be further elucidated.

In COPD patients in this study, the proportion of fibres that stained positive for $\mathrm{COX}$ and $\mathrm{SDH}$ declined in the same order of magnitude as the percentage of type-I fibres. In fact, the majority of the $\mathrm{COX}+$ and $\mathrm{SDH}+$ fibres were classified as type-I fibres, which is underscored by the strong correlation between their proportions. Thus overall, the relative loss of type-I fibres and the loss of oxidative enzyme activity are linked. It has never been established before that the decline in both percentages of fibre type I and the activity of enzymes involved in oxidative energy metabolism in COPD are linked. Since enzyme activity staining and fibre-type classification in this study were performed on serial crosssections, it was not only possible to examine the relationship between the fibre-type redistribution and enzyme activity, but also enzyme activities within the fibre-type categories.

The proportion of $\mathrm{COX}+$ and $\mathrm{SDH}+$ fibres within each of the individual CMM fibre types, I/IIA and IIA, were significantly lower for COPD compared to controls (table 3 ). In addition, the proportion of $\mathrm{COX} \pm$ fibres was higher in CMM type-IIA fibres of patients. It is therefore feasible that $\mathrm{COX}+$ fibres transformed into $\mathrm{COX} \pm$ fibres. It is possible that this extra loss of oxidative capacity is specific for type-IIA fibres. Another possibility is that the loss of oxidative enzyme activity occurs in an early stage of fibretype transition. It is feasible that as a result of the disease (i.e. hypoxia), oxidative energy metabolism is disturbed and the activities of the enzymes involved are reduced. Type-I and IIA fibres strongly depend on aerobic metabolism, whereas in type-IIX fibres energy conversion is based mainly on anaerobic, glycolytic metabolism. It is therefore tempting to state that in type-I fibres the co-expression of the MyHC-IIA isoform and that in type-IIA fibres the co-expression of the MyHC-IIX isoform is an adaptation to impaired oxidative energy metabolism. Alternatively, it is possible that either the reduced oxidative capacity is secondary to the changes in MyHC-isoform contents or that one or more common factors affect both oxidative capacity and MyHC-isoform contents simultaneously.

The present results indicate that the low fibre type-I proportion in COPD is more pronounced in Emph+ patients (stratified by HRCT) compared to Emphpatients. Like others the authors found a positive relationship between fibre type-I proportion and age in healthy subjects [28-30]. Due to the relative loss of type-I fibres this relationship is disturbed in COPD patients, being totally absent in the Emph+. The proportion of $\mathrm{COX}+$ fibres was also lower in Emph+, but not statistically significant. A positive correlation between the $\mathrm{Pa}_{\mathrm{a}} \mathrm{O}_{2}$ and the percentage of type-I fibres has been reported previously $[5,6]$. In addition, a positive relationship between the MyHC-I content, and the $D \mathrm{~L}, \mathrm{CO}$, and the FEV1, has been shown $[10,11]$. However, in the present study these relationships between fibre-type proportions and lung function data were not observed, suggesting that fibre type-I proportion is not related to disease severity per se. COPD patients with a $D \mathrm{~L}, \mathrm{CO}<55 \%$ predicted are likely to desaturate during exercise [31]. Since the $D$ L,CO is lower in the Emph+ patients, it can be speculated that chronic and especially intermittent hypoxia is directly or indirectly involved in causing the lower fibre type-I proportion compared to Emphpatients. This issue requires further investigation.

At this point, it is not completely understood which factors are causing the muscular abnormalities in COPD. Hypoxia has already been discussed earlier. However, oxidative stress may also play a role in the loss of mitochondrial enzyme activity and relative loss of type-I fibres, since it has been demonstrated that mitochondrial proteins are highly susceptible to oxidative damage [32]. The possible role of malnutrition is demonstrated by animal studies in which nutritional deprivation caused depressed muscular mitochondrial capacity [33-35]. Physical inactivity or disuse of limb muscles are potential contributors to the observed fibre-type shift [36]. However, the authors have not observed previously any difference between the daily physical activity levels of Emph+ and Emph- patients [37], while differences in fibre type-I proportions between these subgroups have now been found. In addition, none of the patients in this study was bedridden and it is therefore unlikely that disuse alone caused the very low fibre type-I proportions that were observed.

To conclude, this study demonstrates the involvement of increased proportions of hybrid I/IIA and IIA/IIX fibre types in the fibre-type shift from I to IIX in the vastus lateralis of chronic obstructive pulmonary disease patients compared to controls. The abnormal fibre type-I proportion is aggravated in emphysema patients. In addition, the percentage of fibres with high oxidative enzyme activities were also reduced in chronic obstructive pulmonary disease patients, being closely linked to the reduced fibre type-I proportion. Moreover, despite comparable IIA fibre-type proportions between patients and controls, oxidative capacity is reduced in this fibre type in patients. Further research should be carried out to identify the factors involved and the mechanisms underlying the muscular alterations in order to evaluate current treatment approaches and to develop new approaches.

Acknowledgements. The authors would like to thank A.F.M. Moorman for providing the antibodies against MyHC isoforms I, IIA and IIA+IIX. 


\section{References}

1. Schols AM, Mostert R, Soeters PB, Wouters EF. Body composition and exercise performance in patients with chronic obstructive pulmonary disease. Thorax 1991; 46: 695-699.

2. Cotes JE, Zejda J, King B. Lung function impairment as a guide to exercise limitation in work-related lung disorders. Am Rev Respir Dis 1988; 37: 1089-1093.

3. Gosker HR, Wouters EF, van Der Vusse GJ, Schols AM. Skeletal muscle dysfunction in chronic obstructive pulmonary disease and chronic heart failure: underlying mechanisms and therapy perspectives. $\mathrm{Am}$ $J$ Clin Nutr 2000; 71: 1033-1047.

4. Whittom F, Jobin J, Simard PM, et al. Histochemical and morphological characteristics of the vastus lateralis muscle in patients with chronic obstructive pulmonary disease. Med Sci Sports Exerc 1998; 30: $1467-1474$

5. Jakobsson P, Jorfeldt L, Brundin A. Skeletal muscle metabolites and fibre types in patients with advanced chronic obstructive pulmonary disease (COPD), with and without chronic respiratory failure. Eur Respir $J$ 1990; 3: 192-196.

6. Hildebrand IL, Sylvén C, Esbjörnsson M, Hellström $\mathrm{K}$, Jansson E. Does chronic hypoxaemia induce transformations of fibre types? Acta Physiol Scand 1991; 141: 435-439.

7. Maltais F, Simard AA, Simard C, Jobin J, Desgagnes $\mathrm{P}$, LeBlanc P. Oxidative capacity of the skeletal muscle and lactic acid kinetics during exercise in normal subjects and in patients with COPD. Am J Respir Crit Care Med 1996; 153: 288-293.

8. Jakobsson P, Jorfeldt L, Henriksson J. Metabolic enzyme activity in the quadriceps femoris muscle in patients with severe chronic obstructive pulmonary disease. Am J Respir Crit Care Med 1995; 151: 374 377.

9. Sauleda J, García-Palmer F, Wiesner RJ, et al. Cytochrome oxidase activity and mitochondrial gene expression in skeletal muscle of patients with chronic obstructive pulmonary disease. Am J Respir Crit Care Med 1998; 157: 1413-1417.

10. Satta A, Migliori GB, Spanevello A, et al. Fibre types in skeletal muscles of chronic obstructive pulmonary disease patients related to respiratory function and exercise tolerance. Eur Respir J 1997; 10: 2853-2860.

11. Maltais F, Sullivan MJ, LeBlanc P, et al. Altered expression of myosin heavy chain in the vastus lateralis muscle in patients with COPD. Eur Respir $J$ 1999; 13: 850-854.

12. ATS. Standards for the diagnosis and care of patients with chronic obstructive pulmonary disease (COPD) and asthma. Am Rev Respir Dis 1987; 136: 225-244.

13. Quanjer P, Tammeling GJ, Cotes JE, Pedersen OF, Peslin R, Yernault JC. Standardized lung function testing. Eur Respir J 1993; 6: 5-40.

14. ATS. Chronic bronchitis, asthma, and pulmonary emphysema by the committee on diagnostic standards for nontubercolosis respiratory disease. Am Rev Respir Dis 1962; 85: 762-812.

15. Lamers RJ, Thelissen GR, Kessels AG, Wouters EF, van Engelshoven JM. Chronic obstructive pulmonary disease: evaluation with spirometrically controlled CT lung densitometry. Radiology 1994; 193: 109-113.

16. Sakai F, Gamu G, Im JG, Ray CS. Pulmonary function abnormalities in patients with CT-determined emphysema. J Comput Assist Tomogr 1987; 11: $963-$ 968.

17. Bergstrom L. Muscle electrolytes in man. Determination by neutron activation analysis on needle biopsy specimens. A study on normal subjects, kidney patients, and patients with chronic diarrhea. Scand J Clin Lab Invest 1962; 68: 1-110.

18. Dammeijer PF, van Mameren $\mathrm{H}$, van Dijk $\mathrm{P}$, et al. Stapedius muscle fibre composition in the rat. Hear Res 2000; 141: 169-179.

19. Ogilvie RW, Feeback DL. A metachromatic dyeATPase method for the simultaneous identification of skeletal muscle fiber types I, IIA, IIB and IIC. Stain Technol 1990; 65: 231-241.

20. Guth L, Samaha FJ. Procedure for the histochemical demonstration of actomyosin ATPase. Exp Neurol 1970; 28: 365-367.

21. Sant'Ana Pereira JA, Ennion S, Sargeant AJ, et al. Comparison of the molecular, antigenic and ATPase determinants of fast myosin heavy chains in rat and human: a single-fibre study. Pflügers Arch 1997; 435: 151-163.

22. Brooke MH, Kaiser KK. Muscle fiber types: how many and what kind? Arch Neurol 1970; 23: 369-379.

23. Sant'Ana Pereira JA, Wessels A, Nijtmans L, Moorman AF, Sargeant AJ. New method for the accurate characterization of single human skeletal muscle fibres demonstrates a relation between mATPase and MyHC expression in pure and hybrid fibre types. J Muscle Res Cell Motil 1995; 16: 21-34.

24. Pearse AG. Histochemistry: Theoretical and applied. 3rd edition. Edinburgh, Churchill Livingstone, 1972.

25. Sheehan DC, Hrapchak BB. Theory and practice of histotechnology. 2nd edition. Columbus, Battelle Memorial Institute, 1987.

26. Altman DG, Gore SM, Gardner MJ, Pocock SJ. Statistical guidelines for contributors to medical journals. Br Med J Clin Res Ed 1983; 286: 1489-1493.

27. Wang XN, Williams TJ, McKenna MJ, et al. Skeletal muscle oxidative capacity, fiber type, and metabolites after lung transplantation. Am J Respir Crit Care Med 1999; 160: 57-63.

28. Larsson L, Grimby G, Karlsson J. Muscle strength and speed of movement in relation to age and muscle morphology. J Appl Physiol 1979; 46: 451-456.

29. Klitgaard H, Mantoni M, Schiaffino S, et al. Function, morphology and protein expression of ageing skeletal muscle: a cross-sectional study of elderly men with different training backgrounds. Acta Physiol Scand 1990; 140: 41-54.

30. Poggi P, Marchetti C, Scelsi R. Automatic morphometric analysis of skeletal muscle fibers in the aging man. Anat Rec 1987; 217: 30-34.

31. Owens GR, Rogers RM, Pennock BE, Levin D. The diffusing capacity as a predictor of arterial oxygen desaturation during exercise in patients with chronic obstructive pulmonary disease. N Engl J Med 1984; 310: 1218-1221.

32. Haycock JW, Jones P, Harris JB, Mantle D. Differential susceptibility of human skeletal muscle proteins to free radical induced oxidative damage: a histochemical, immunocytochemical and electron microscopical study in vitro. Acta Neuropathol (Berl) 1996; 92: 331-340.

33. Sieck GC, Lewis MI, Blanco CE. Effects of undernutrition on diaphragm fiber size, SDH activity, 
and fatigue resistance. J Appl Physiol 1989; 66: 21962205.

34. Russell DM, Atwood HL, Whittaker JS, et al. The effect of fasting and hypocaloric diets on the functional and metabolic characteristics of rat gastrocnemius muscle. Clin Sci 1984; 67: 185-194.

35. Madapallimattam A, Law L, Jeejeebhoy K. Effect of hypocaloric feeding on mitochondrial respiratory chain activity. Clin Nutr 2000; 19: 23.
36. Burnham R, Martin T, Stein R, Bell G, MacLean I, Steadward R. Skeletal muscle fibre type transformation following spinal cord injury. Spinal Cord 1997; 35: 86-91.

37. Engelen MPKJ, Schols AMWJ, Does JD, Gosker HR, Deutz NEP, Wouters EFM. Exercise induced lactate increase in relation to muscle substrates in patients with COPD. Am J Respir Crit Care Med 2000; 162: 1697-1704. 